\title{
Use of paper selectively absorbing long wavelengths to reduce the impact of educational near work on human refractive development
}

\author{
Ronald H H Kröger, Stefanie Binder
}

\begin{abstract}
Backgroundlaims-Educational near work has been identified as a major risk factor for the development of juvenile progressive myopia. A study was undertaken to determine whether differences in focal length resulting from longitudinal chromatic aberration of the eye can be exploited to reduce the impact of near work on refractive development.

Methods-Infrared photorefraction was used to determine refractive states in young adult volunteers performing a task similar to reading and writing under various spectral environments. The potential benefits of the observed differences in accommodation demand were studied with a computational model of emmetropisation and myopia progression.

Results-The refractive state was largely independent of the colour temperature of the illumination light (white paper) and the colour of commercially available papers (white illumination). Selective elimination of long wavelengths, however, significantly reduced the accommodation stimulus by about 0.5 dioptres.

Conclusion-Results from model calculations suggest that the use of paper which selectively absorbs long wavelengths may significantly reduce the myopiagenic effects of educational near work. (Br F Ophthalmol 2000;84:890-893)
\end{abstract}

In the myopic eye images of distant objects are created in front of the retina even when accommodation is fully relaxed, since the bulbus is too long because of excessive axial growth. ${ }^{1}$ The refractive state of the human eye at birth shows considerable variation between individuals. Eye size is usually adjusted to mild hyperopia within the first year of life (emmetropisation). ${ }^{2}$ In western societies the prevalence of myopia in 6 year old children is only about $2 \%{ }^{3}$ compared with $20-60 \%$ in the adult population. ${ }^{4}$ Educational near work such as reading and writing is a major risk factor since myopia usually develops during school years, ${ }^{2}$ is positively correlated with educational status, ${ }^{5}$ starts earliest resulting in most myopic adult refractions in nations with rigorous educational systems, ${ }^{6}$ and is almost unknown in societies with no formal education. ${ }^{7}$ Even individuals with a genetic predisposition for myopia usually become emmetropic or slightly hyperopic in indigenous populations. ${ }^{8}$ If a child becomes myopic, the refractive error usually increases (myopia progression) until adulthood is reached. ${ }^{9}$ In animal models of myopia imposed refractive error is compensated by appropriate changes in eye growth, indicating that the growth of the vertebrate eye is under the control of visual feedback. This guidance of eye growth appears to generate myopiagenic signals during periods of extensive near work.

The risk of developing myopia is correlated with near point esophoria and the onset of myopia usually coincides with an eso shift in near phoria. ${ }^{10}$ To avoid double images, children with near point esophoria have to underaccommodate when reading or writing. The resulting image blur is assumed to stimulate eye growth. ${ }^{11}$ Measures that reduce the accommodation stimulus, but not the convergence demand, improve the match between accommodation and convergence and should therefore be effective in preventing myopia and/or slowing its progression. Since the focal length of the eye decreases with decreasing wavelength of light (longitudinal chromatic aberration, LCA), we experimentally determined the reduction of accommodation demand achievable by varying the spectral environment during near work. The potential benefits for refractive development were studied in model calculations.

\section{Materials and methods}

Young volunteers (20-28 years) wearing their best subjective corrections of refractive error, if present, fixated a black cross printed on paper at a distance of $33 \mathrm{~cm}$, requiring 3 dioptres of accommodation of the emmetropic eye. The paper was illuminated with a laboratory halogen lamp (Zeiss). In a first set of measurements the colour temperature was varied between approximately $1300 \mathrm{~K}$ (candle light), $2300 \mathrm{~K}$ (incandescent light), and $3300 \mathrm{~K}$ (halogen light) by controlling the voltage supplied to the lamp; light of about $5500 \mathrm{~K}$ was produced with a daylight filter (Zeiss). The fixation target was printed on white paper and illuminances were adjusted to 22 lux with neutral grey (NG) filters. In a second series of experiments we used coloured papers to restrict the spectrum available to the eye. The targets were illuminated with $3300 \mathrm{~K}$ of constant intensity (22 lux with white paper). Red (and yellow) papers usually have long pass (LP) characteristics - that is, they reflect only long (and middle, respectively) wavelengths. Commercially available green and blue papers have more complicated reflectances (Fig 1A). In a third series of measurements we therefore 

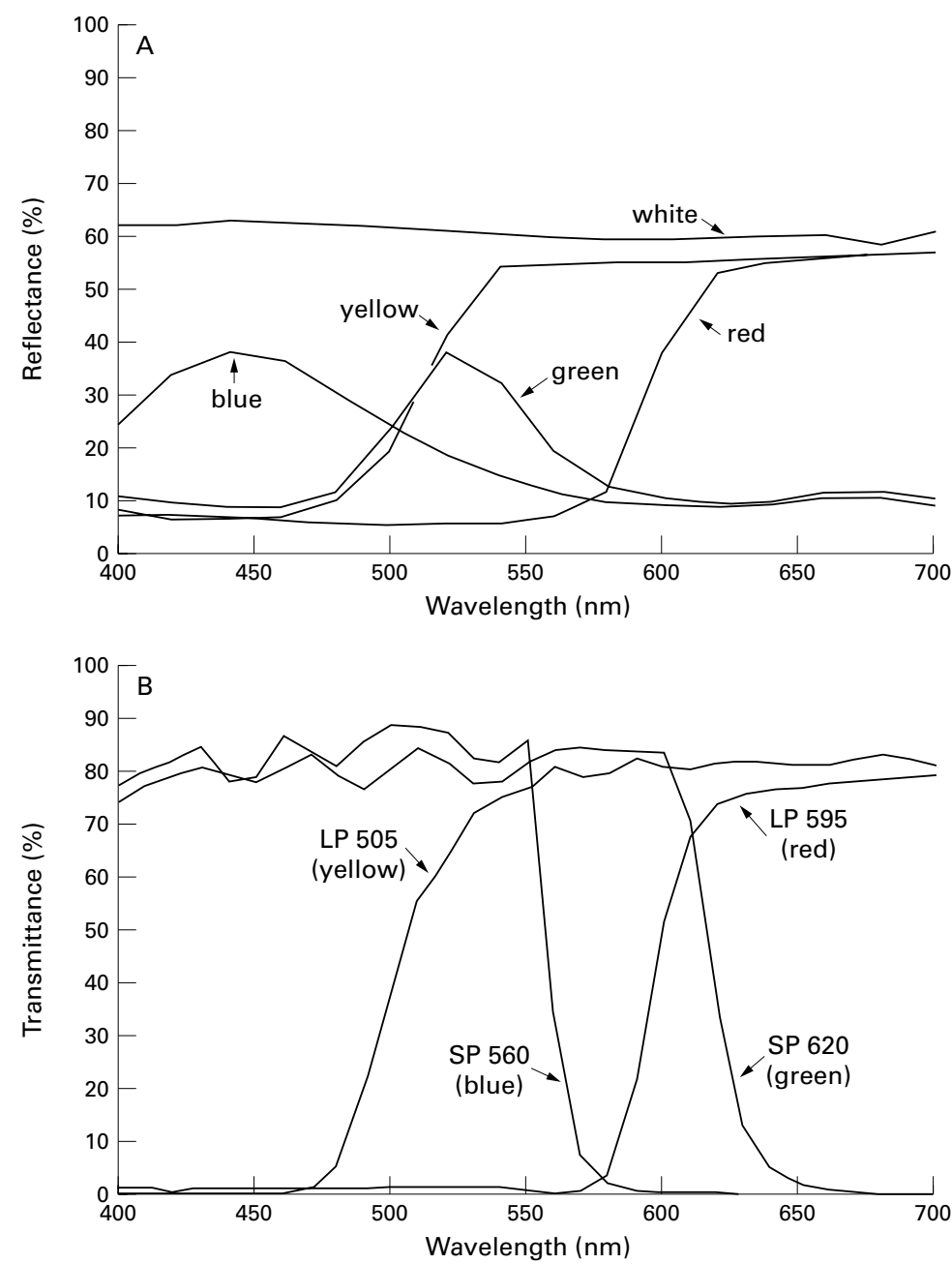

Figure 1 Paper reflectances and filter transmittances. (A) The white paper used had about the same reflectivity throughout the visible spectrum. The red and yellow papers had long pass characteristics with half maximum cut off values at $590 \mathrm{~nm}$ and $510 \mathrm{~nm}$, respectively. The green and blue papers had band pass characteristics, low total reflectance, and also reflected long wavelengths to some extent. (B) The selected long pass filters had similar cut off values as the papers of matching colours. The short pass filters had sharp cut off values and high transmittances for short wavelengths.

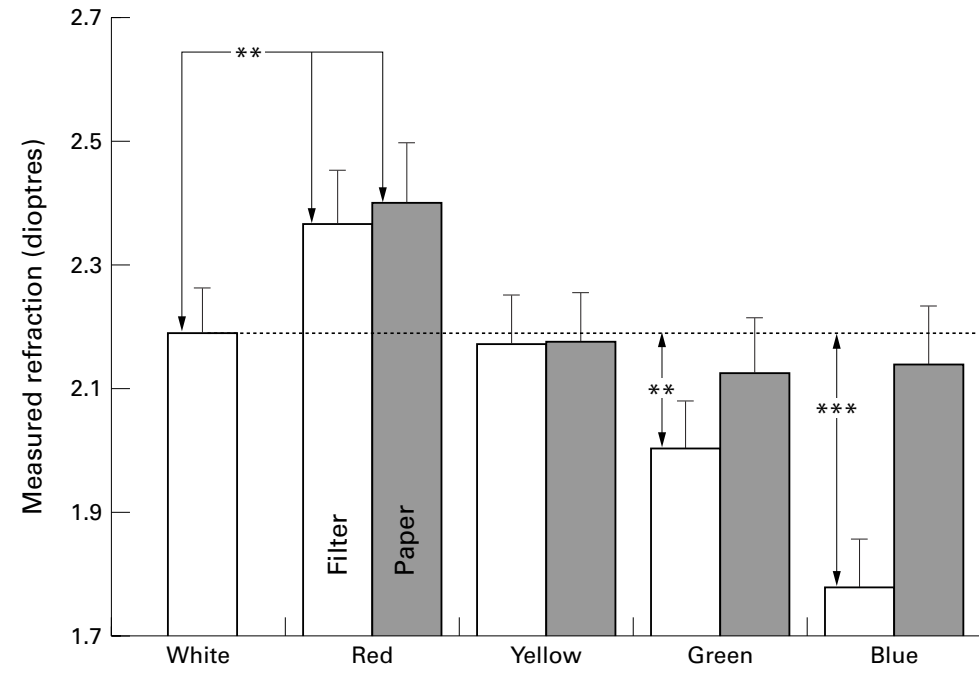

Figure 2 Effect of long pass and short pass filters on the refractive state of the eye during a visual task similar to reading and writing. The red and yellow papers and filters had similar long pass characteristics. Accordingly, accommodative effort was almost identical with papers and filters of the same colour. Standard green and blue papers with band pass characteristics had no effect on refractive state. ${ }^{\star \star} p<0.01,{ }^{\star \star *} p<0.001$, paired t test. used interference edge filters (Andover) in the illumination pathway in combination with white paper to simulate short pass (SP) paper reflectance characteristics (Fig 1B). LP foil filters (Lee) with transmittances similar to the reflectances of yellow and red papers (Fig 1) were used for comparisons. Illuminances with the LP filters in the illumination pathway combined with white paper were adjusted with an NG filter to the values measured with yellow and red papers illuminated with white light. The same NG filter was combined with the SP filters to account for the loss in brightness expected if equivalently pigmented papers were used in educational near work.

Paper reflectances were measured with a Shimadzu UV2101-PC spectrophotometer fitted with an integrating sphere (Shimadzu ISR260). Filter transmittances were determined with a Beckman DU 640 spectrophotometer. Refractive states were measured by slope based eccentric infrared photorefraction ${ }^{12}$ through an infrared transmitting port just underneath the fixation target. The measurement device was calibrated for each individual using ophthalmic lenses from +0.5 to +2.5 dioptres with relaxed accommodation in the dark. The sequences of papers and filters of different colours were randomised for each subject.

The potential effects of different illuminations and paper colours were studied with Flitcroft's computational model of emmetropisation and myopia progression. ${ }^{11}$ Except for emmetropisation gain, which determines the rate of change, the parameters used in model calculations (see Appendix) were based on measurements in myopic and emmetropic human subjects. ${ }^{11} 13$

\section{Results}

The effects of different colour temperatures of illumination light were measured in 40 individuals. Between 1300 and $5500 \mathrm{~K}$ there was only a small (<0.2 dioptres) statistically insignificant reduction in the accommodative response.

Blue and green papers had no significant effect on the refractive state in 20 volunteers (Fig 2). Inserting SP filters into the illumination pathway, however, significantly reduced accommodative responses by 0.41 ("blue", SP $560, \mathrm{p}<0.001$, paired $t$ test) and 0.19 ("green", SP 620, p<0.01) dioptres. Red and yellow papers had about the same effects as the corresponding LP filters (Fig 2).

The measured refractions were lower than the actual values because of tonic accommodation in the dark, which biased the calibration of the measuring device. Assuming that the subjects accommodated 2.8 dioptres at the 3 dioptre stimulus when white light and paper were used, the corrected reduction of accommodation demand achieved with the SP 560 filter was 0.52 dioptres. Under conditions of extensive near work ( $80 \%$ of viewing time) at a distance of $33 \mathrm{~cm}$, model calculations predict that myopes would develop -1.11 dioptres instead of -1.38 dioptres of myopia if the accommodation stimulus during near work is reduced by 0.5 dioptres and no distance 
correction is used. If myopes are corrected when they reach a difference of -0.5 dioptres between the currently worn distance correction and the refractive error of the eye, the refractive state does not stabilise and susceptible individuals acquire -4.70 dioptres of myopia after 100 iterations of the model, which we assumed to be equivalent to the age when eye growth stops (adult refraction). With distance corrections, reduction of the accommodation stimulus during near work by 0.5 dioptres would reduce the adult refractive error to -3.50 dioptres.

\section{Discussion}

The small effects on the refractive state of differences in colour temperature suggest that the visual system primarily uses long wavelengths, if available, during reading tasks. The precise spectral composition of the illumination light therefore seems to be of little relevance, provided that long wavelengths are present. The blue and green papers available to us had band pass characteristics, low total reflectivity, and reflected long wavelengths to some extent (Fig 1). Consequently, accommodation demand was not significantly reduced. This was achieved, however, by selective removal of long wavelengths from the light available to the eye (Fig 2).

No discomfort or problems with keeping fixation steady were reported when long wavelengths were removed with SP filters, which were almost colourless (SP 620) or of pale blue colour (SP 560). Reducing spectral band width with SP filters thus had no apparent effect on normal visual function, probably because chromatic aberration in the short to middle wave range of the spectrum could still be used to drive accommodation.

It seems to be irrelevant whether the spectrum of light is restricted by pigmented papers or by filters in the illumination pathway, since there were close agreements between the results obtained with red and yellow papers and the corresponding LP filters. Our results therefore suggest that the use of paper which selectively absorbs long wavelengths in a similar range to the SP 560 filter may be effective in reducing the myopiagenic effects of educational near work. Unfortunately, an extensive search for such paper was unsuccessful. Because of the light colours of pigments with suitable characteristics, they may not be useful for the manufacture of coloured paper. This does not preclude, however, that such pigments exist.

Efforts to slow the progression of myopia in children have included long term topical administration of cycloplegic agents that reduce or block accommodative ability. How- ever, the side effects were severe or the effects on refractive development were small. ${ }^{14} \mathrm{Re}$ cently, a large prospective longitudinal study has been launched ${ }^{15}$ to investigate the potency of bifocal spectacles to arrest the progression of juvenile myopia. Bifocals correct near point esophoria in a similar way to the removal of long wavelengths and appear to be effective in slowing the progression of myopia. ${ }^{16-19}$ Spectacles are worn, however, when myopia has already developed and can only slow or halt its progression. In contrast, the use of long wave absorbing paper may prevent or at least delay the onset of myopia in children at low to moderate risk of developing myopia, and may slow the progression in individuals at high risk, even before distance correction is required.

The authors thank M Ott for his help with photorefractive measurements, R H Douglas for the measurements of paper reflectances, H-J Wagner, $M$ Ott and B Hirt for useful discussions, and all volunteers for their cooperation.

Funding: Supported by DFG Wa 348/17.

1 Wildsoet CF. Active emmetropization: evidence for its existence and ramifications for clinical practice. Ophthal Physiol Optics 1997;17:279-90.

2 Gwiazda J, Thorn F, Bauer J, et al. Emmetropization and the progression of manifest refraction in children followed progression of manifest refraction in children foll
from infancy to puberty. Clin Vis Sci 1993;8:337-44.

3 Mutti DO, Zadnik K, Adams AJ. Myopia: the nature versus nurture debate goes on. Invest Ophthalmol Vis Sci 1996;37: $952-7$.

4 Framingham Offspring Eye Study Group. Familial aggregation and prevalence of myopia in the Framingham Offspring Eye Study. Arch Ophthalmol 1996;114:326-32.

5 Katz J, Tielsch JM, Sommer A. Prevalence and risk factors for refractive errors in an adult inner city population. Invest Ophthalmol Vis Sci 1997;38:334-40.

6 Ho PCP, Lo PI, Lau J. The significance of the age of onset of myopia among university students of the Chinese race. Invest Ophthalmol Vis Sci (Suppl) 1995;36:S1073.

7 Thorn F, Cruz AAV, Machado AJ, et al. Refractive status of the indigenous people of the upper Amazon basin. Invest Ophthalmol Vis Sci 1998;39:S898.

8 Norn M. Myopia among the Inuit population of East Greenland. Longitudinal study 1950-1994. Acta Ophthalmol Scand 1997;75:723-5.

9 Curtin BJ. The myopias: basic science and clinical management. New York: Harper and Row, 1985.

10 Goss DA, Jackson TW. Clinical findings before the onset of myopia in youth: 3. Heterophoria. Optom Vis Sci 1996;73: 269-78.

11 Flitcroft DI. A model of the contribution of oculomotor and optical factors to emmetropization and myopia. Vis Res 1998;38:2869-79.

12 Schaeffel F, Wilhelm H, Zrenner E. Inter-individual variability in the dynamics of natural accommodation in humans: relation to age and refractive errors. $\mathcal{F}$ Physiol 1993;461:301-20.

13 Hung GK, Ciuffreda KJ, Rosenfield M. Proximal contribution to a linear static model of accommodation and vergence. Ophthal Physiol Optics 1996;16:31-41.

14 Yen M, Liu JH, Kao S, et al. Comparison of the effect of atropine and cyclopentolate on myopia. Ann Ophthalmol atropine and cyclo

15 Fulk GW, Cyert LA, Parker DE. Baseline characteristics in the Myopia Progression Study, a clinical trial of bifocals to slow myopia progression. Optom Vis Sci 1998;75:485-92.

16 Goss DA. Variables related to the rate of childhood myopia progression. Optom Vis Sci 1990;67:631-6.

17 Goss DA, Grosvenor T. Rates of childhood myopia progression with bifocals as a function of nearpoint phoria: consistency of three studies. Optom Vis Sci 1990;67:63740.

18 Goss DA. Effect of spectacle correction on the progression of myopia in children: a literature review. $\mathcal{F}$ Am Optom Ass 1994;65:117-28.

19 Fulk GW, Cyert LA. Can bifocals slow myopia progression? f Am Optom Ass 1996;67:749-54. 


\section{Appendix}

\begin{tabular}{|c|c|c|c|}
\hline Parameters of the model ${ }^{11}$ & $\begin{array}{l}\text { Low risk } \\
\text { (emmetropes) }\end{array}$ & $\begin{array}{l}\text { High risk } \\
\text { (myopes) }\end{array}$ & \\
\hline Accommodation gain $(\mathrm{Ag})$ & 10 & 3 & \\
\hline Vergence gain $(V g)$ & 150 & 150 & \\
\hline Accommodative convergence per unit of accommodation (AC/A ratio, $A C$ ) & 0.8 & 1.2 & Metre angles per dioptre \\
\hline Convergence accommodation per unit of convergence $(\mathrm{CA} / \mathrm{C}$ ratio, $C A)$ & 0.37 & 0.2 & Dioptres per metre angle \\
\hline Tonic accommodation $(A t)$ & 0.61 & 0.25 & Dioptres \\
\hline Tonic vergence (phoria, $V t$ ) & 0.29 & 2 & Metre angles \\
\hline Emmetropisation gain $(E g)$ & 0.1 & 0.1 & \\
\hline Tnear/Tfar & $80 / 20$ & $80 / 20$ & $\%$ \\
\hline$A s / V s$ (white paper) & $3.0 / 3.0$ & $3.0 / 3.0$ & Dioptres/metre angle \\
\hline$A s / V s$ (long wave absorbing paper) & $2.5 / 3.0$ & $2.5 / 3.0$ & Dioptres/metre angle \\
\hline
\end{tabular}

